\section{Incidence and risk factors for traumatic intraocular pressure elevation and traumatic glaucoma after open-globe injury}

KD Bojikian, AL Stein, MA Slabaugh and PP Chen

\begin{abstract}
Purpose To examine traumatic intraocular pressure (IOP) elevation and glaucoma after open-globe injury.

Design Retrospective, observational case series. Methods Review of patients with open-globe repair at the University of Washington from May 1997 through July 2010. Traumatic IOP elevation and glaucoma were defined respectively as intraocular pressure (IOP) $\geq 22 \mathrm{~mm} \mathrm{Hg}$ at $>1$ visit or need for glaucoma medication, and long-term (at least 3 months) glaucoma medication use or glaucoma surgery.
\end{abstract} Results We included 515 eyes (515 patients). The mean follow-up was $12.6 \pm 20.1$ months. One hundred twenty eyes $(23.3 \%)$ developed traumatic IOP elevation, of which $32(6.2 \%)$ developed glaucoma; six eyes $(1.2 \%)$ required glaucoma surgery. The mean time to development of traumatic IOP elevation was $1.5 \pm 3.4$ months (range 1 day to 2 years). Kaplan-Meier 6- and 12-month estimates for development of traumatic IOP elevation were 27.2 and $32.4 \%$, respectively, and for development of traumatic glaucoma were 7.1 and $\mathbf{1 1 . 0} \%$, respectively. Multivariate regression revealed associations between traumatic IOP elevation and older age, and traumatic glaucoma and prior penetrating keratoplasty, initial vitreous hemorrhage, Zone II injury, and penetrating keratoplasty after open-globe repair. Traumatic glaucoma was controlled (IOP $<22 \mathrm{~mm} \mathrm{Hg}$ ) in $78.1 \%$ of eyes at final follow-up, with mean IOP of $18.2 \mathrm{~mm} \mathrm{Hg}$ on 1.7 medications.

Conclusions Traumatic IOP elevation and glaucoma were common after visually salvageable open-globe injury. Most cases developed within 6 months, although longer follow-up remains important for case detection. Penetrating keratoplasty before or after repair, and vitreous hemorrhage were notable risk factors.

Eye (2015) 29, 1579-1584; doi:10.1038/eye.2015.173; published online 18 September 2015

\section{Introduction}

Eye trauma resulting in an open globe is an important public health problem, with an estimated global incidence for 2013 of 250000 cases (3.5/100 000 persons per year). ${ }^{1}$ Traumatic IOP elevation and traumatic glaucoma are complications that can result from the initial eye injury and surgical repair, and may require further treatment. ${ }^{2}$ Causes of IOP elevation after trauma include trabecular meshwork damage, hyphema, injury to the lens and/or iris, inflammation, peripheral anterior synechiae, vitreous hemorrhage, and topical corticosteroid use. ${ }^{2-5}$ Several studies have examined traumatic IOP elevation or glaucoma following closed (non-surgical) globe injury. ${ }^{5-7}$ However, few large studies have evaluated IOP elevation and traumatic glaucoma after open-globe injury. We sought to evaluate the prevalence and potential risk factors for traumatic IOP elevation and glaucoma after open-globe injury over a 13-year period at the University of Washington.

\section{Materials and methods}

This study was approved by the Human Subjects Division of the University of Washington. We reviewed patients seen between May 1997 and July 2010 at the Eye Clinics of the University of Washington (University of Washington Medical
Department of Ophthalmology, University of Washington, Seattle, WA, USA

Correspondence: KD Bojikian,

Ophthalmology, University of Washington, 325 Ninth Ave, Box 359608, Seattle, WA 98104-2499, USA Tel: +1 206744 2020; Fax: +1 2066857055 . E-mail: bkarine@uw.edu

Received: 29 May 2014 Accepted in revised form: 15 July 2015

Published online: 18 September 2015 
Center and Harborview Medical Center). Patients who underwent surgical repair of open-globe injury at the University of Washington were identified with International Classification of Disease-9 codes of 871, 871.1, 871.2, 871.9, and Current Procedural Terminology codes 65270-65290. We excluded patients who required enucleation for an irreparably traumatized eye within 3 months after trauma, or who had a previous diagnosis of glaucoma, and patients younger than 12 years old. When both eyes of one patient were eligible, the right eye was chosen for the study.

Clinical data recorded included basic demographics (age, sex, and race), mechanism (blunt vs sharp) and location of injury, visual acuity (VA) at presentation, presence of relative afferent pupillary defect (RAPD), hyphema, lens injury, vitreous hemorrhage, presence of

Table 1 Baseline and clinical characteristics of the population studied $(N=515$ eyes $)$

\begin{tabular}{|c|c|}
\hline Age (years) & $42.4 \pm 20.3$ (range 12-96) \\
\hline Follow-up (months) & $12.6 \pm 20.1$ (median 4.0 ) \\
\hline \multicolumn{2}{|l|}{ Sex } \\
\hline Male & $396(76.9 \%)$ \\
\hline Female & $119(23.1 \%)$ \\
\hline \multicolumn{2}{|l|}{ Race } \\
\hline Caucasian & $353(68.5 \%)$ \\
\hline Hispanic & $81(15.7 \%)$ \\
\hline African American & $39(7.6 \%)$ \\
\hline Other/not listed & $42(8.2 \%)$ \\
\hline \multicolumn{2}{|c|}{ Initial LogMAR visual acuity ( $N=472$ eyes) } \\
\hline $3.00(\mathrm{NLP})$ & $32(6.8 \%)$ \\
\hline $2.70(\mathrm{LP})$ & $169(35.8 \%)$ \\
\hline $2.30(\mathrm{HM})$ & $66(14.0 \%)$ \\
\hline $1.00-1.90(20 / 200-C F)$ & $106(22.4 \%)$ \\
\hline $0.50-0.90(20 / 63-20 / 160)$ & $31(6.7 \%)$ \\
\hline$<0.50$ & $68(14.3 \%)$ \\
\hline \multicolumn{2}{|l|}{ Object causing injury } \\
\hline Sharp & $286(55.5 \%)$ \\
\hline Blunt & $229(44.5 \%)$ \\
\hline \multicolumn{2}{|c|}{ Ocular Trauma Score grade ( $N=466$ eyes) } \\
\hline 1 & $103(22.1 \%)$ \\
\hline 2 & $196(42.0 \%)$ \\
\hline 3 & $125(26.8 \%)$ \\
\hline 4 & $42(9.1 \%)$ \\
\hline 5 & $0(0 \%)$ \\
\hline \multicolumn{2}{|c|}{ Final LogMar visual acuity ( $N=493$ eyes) } \\
\hline $3.00(\mathrm{NLP})$ & $44(9.0 \%)$ \\
\hline $2.70(\mathrm{LP})$ & $70(14.2 \%)$ \\
\hline $2.30(\mathrm{HM})$ & $69(14.0 \%)$ \\
\hline $1.00-1.90(20 / 200-C F)$ & $99(20.0 \%)$ \\
\hline $0.50-0.90(20 / 63-20 / 160)$ & $32(6.5 \%)$ \\
\hline$<0.50$ & $179(36.3 \%)$ \\
\hline
\end{tabular}

Results given as mean \pm SD where applicable. intraocular foreign body, endophthalmitis, previous ocular disease, subsequent surgical procedures performed, IOP at all follow-up visits, and final VA. We noted the need for any medical or surgical treatment to reduce IOP.

Traumatic IOP elevation was defined as IOP $\geq 22 \mathrm{~mm} \mathrm{Hg}$ in the affected eye at $>1$ visit, or need for treatment of elevated IOP. ${ }^{8}$ Traumatic glaucoma was defined as long-term glaucoma medication use (at least 3 months) or need for glaucoma surgery. ${ }^{5,9}$ All Snellen VA measurements were converted to logarithm of the minimum angle of resolution (logMAR) for statistical analysis. LogMAR values used for low-vision measurements were 1.90 for counting fingers, 2.30 for hand motion, 2.70 for light perception, and 3.00 for no light perception. ${ }^{10}$ Different values were assigned for light perception and no light perception due to the importance of light perception for physiologic functions that are dependent on normal circadian rhythm. ${ }^{11,12}$

All open-globe injuries were categorized by the Open Globe Classification System into three anatomical zones. ${ }^{13}$ Zone I includes the cornea and limbus, Zone II is located in the sclera up to $5 \mathrm{~mm}$ posterior to the limbus, and Zone III, which includes the macula and optic nerve, is posterior to Zone II. The Ocular Trauma Score (OTS $)^{14}$ was used retrospectively to grade the severity of the open-globe injury. In brief, the OTS score is a simplified categorical system for standardized assessment and visual prognosis based on one functional (initial VA) and five anatomical characteristics: rupture, endophthalmitis, perforating injury, retinal detachment, and RAPD. Each variable receives a raw point value, and the sum of raw points is converted into the OTS value of $1-5$; lower numbers indicate more severe injury.

Data were entered into a spreadsheet (SPSS software version 16.0 for Mac; SPSS, Inc., Chicago, IL, USA). Statistical analysis was performed with Kaplan-Meier survival analysis, and univariate and multivariate Cox proportional hazards regression. Risk factors found to have $P \leq 0.2$ in univariate analysis were included in the multivariate analysis.

\section{Results}

We recorded data from 515 eyes of 515 patients who met inclusion criteria. Demographic data, patient characteristics, and OTS classification are presented in Table 1. Most patients were male (76.9\%) and white $(68.5 \%)$, with mean age of $42.4 \pm 20.3$ years. The mean and median follow-up was $12.6 \pm 20.1$ and 4.0 months, respectively. In 49 eyes the OTS was not calculated because the initial VA or the presence of RAPD was not recorded. 
One hundred twenty eyes (23.3\%) developed traumatic IOP elevation. The mean time to develop traumatic IOP elevation was $1.5 \pm 3.4$ months (median 0.25 months; range $0.03-23.8$ months); $93 \%$ were diagnosed by 6 months, and $99 \%$ by 12 months. The Kaplan-Meier estimate for development of traumatic IOP elevation was $27.2 \%$ (95\% confidence interval (CI) $22.6-31.8 \%$ ) at 6 months $(n=162)$, and $32.4 \%(95 \%$ CI $27.0-37.8 \%)$ at 12 months $(n=113)$. With univariate Cox proportional hazards regression analysis, age and a worse OTS score (1 or 2) were associated with traumatic IOP elevation; however, multivariate analysis revealed the only risk factor to be age $(P=0.001$; Table 2$)$.

Traumatic glaucoma developed in 32 eyes $(26.7 \%$ of eyes with traumatic IOP elevation, $6.2 \%$ of all eyes). Longterm glaucoma medication use was required in 30 eyes (5.8\% of all eyes). Six eyes (1.2\% of all eyes, $18.8 \%$ of those with traumatic glaucoma) required glaucoma surgery for IOP control. The mean time to initial development of elevated IOP in these patients was $3.8 \pm 5.6$ months (median 0.9 months; range 0.03-23.8 months); 72\% were diagnosed by 6 months, and $91 \%$ by 12 months after repair. The Kaplan-Meier estimate for development of traumatic glaucoma was 7.1\% (95\% CI 4.3-9.9\%) at 6 months $(n=198)$, and $10.1 \%(95 \%$ CI $6.8-15.2 \%)$ at 12 months $(n=137)$. With univariate Cox proportional hazards regression analysis, several risk factors for development of traumatic glaucoma were identified
(Table 3). However, multivariate analysis revealed the following associations: vitreous hemorrhage at the time of injury $(P=0.003)$, Zone II injury $(P=0.028)$, penetrating keratoplasty $(\mathrm{PKP})$ before open globe $(P=0.009)$, and PKP after open-globe repair $(P<0.001$; Tables 3 and 4$)$.

Among six eyes requiring glaucoma surgery, four eyes (66.7\%) underwent Baerveldt tube shunt placement and two eyes $(33.3 \%)$ underwent contact transcleral diode laser cyclophotocoagulation. Five of six patients $(83.3 \%)$ required glaucoma surgery within 21 months of the initial injury. The mean number of intraocular surgeries (including open-globe repair) before glaucoma surgery was 3.0; five eyes had pars plana vitrectomy, four had PKP before $(n=3)$ or concurrently $(n=1)$, and three had scleral buckle surgery. All six eyes that had glaucoma surgery had IOP $\leq 21 \mathrm{~mm} \mathrm{Hg}$ at the last follow-up visit.

Having subsequent PKP after open-globe repair was significantly associated with development of traumatic glaucoma $(P<0.001$; Table 4$)$. Two of 39 patients $(5.1 \%)$ who had PKP before open-globe injury and who then required repeat $\mathrm{PKP}$, developed traumatic glaucoma. If patients who had PKP before open-globe injury are excluded from analysis, the association with glaucoma remains significant (data not shown; $P=0.001$ ). Of nine patients with traumatic glaucoma who did not have PKP before traumatic open-globe injury, five (55.5\%) developed traumatic glaucoma before the subsequent PKP surgery.

Table 2 Univariate Cox proportional hazards regression analysis of risk factors for traumatic IOP elevation after open-globe injury

\begin{tabular}{lccc}
\hline Risk factors & $H R$ & $95 \%$ CI & P-value \\
\hline Age & $1.019 /$ year & $1.010-1.028$ & $<0.001^{\text {a }}$ \\
Prior penetrating keratoplasty & 1.340 & $0.951-1.887$ & 0.095 \\
Initial logMAR visual acuity $^{\mathrm{b}}$ & 1.231 & $0.987-1.536$ & 0.065 \\
Ocular Trauma Score 1 or 2 (worse trauma) $^{\mathrm{c}}$ & 1.307 & $1.051-1.625$ & 0.016 \\
Ocular Trauma Score $^{\mathrm{c}}$ & $0.854 /$ larger & $0.683-1.067$ & 0.164 \\
Vitreous hemorrhage $_{\text {Penetrating keratoplasty after open globe }}$ & 1.172 & $0.976-1.407$ & 0.089 \\
\hline
\end{tabular}

Abbreviations: CI, confidence interval; HR, hazard ratio. ${ }^{a} \mathrm{HR}=1.019 /$ year, $95 \%$ CI 1.007-1.027, $P=0.001$, multivariate Cox proportional hazards regression. ${ }^{\mathrm{b}} n=472$ for $\log$ MAR VA analysis. ${ }^{\mathrm{c}} n=466$ for all OTS analyses.

Table 3 Univariate Cox proportional hazards regression of risk factors for traumatic glaucoma after open globe

\begin{tabular}{lccr}
\hline Risk factors & $H R$ & $95 \%$ CI & P-value \\
\hline Age & $1.017 /$ year & $0.999-1.035$ & 0.069 \\
Prior penetrating keratoplasty & 1.657 & $0.912-3.012$ & 0.098 \\
Initial logMAR visual acuity $^{\mathrm{a}}$ & 1.877 & $1.090-3.230$ & 0.023 \\
Zone II injury $_{\text {Ocular Trauma Score 1 or 2 (worse trauma) }}^{\mathrm{b}}$ & 1.391 & $0.982-1.970$ & 0.063 \\
Ocular Trauma Score $^{\mathrm{b}}$ & 1.871 & $1.105-3.168$ & 0.020 \\
Vitreous hemorrhage $_{\text {Pars plana vitrectomy }}$ & $0.665 /$ larger & $0.425-1.043$ & 0.076 \\
Scleral buckle & 2.234 & $1.386-3.601$ & 0.001 \\
Penetrating keratoplasty after open globe & 1.503 & $1.046-2.160$ & 0.027 \\
\hline
\end{tabular}

Abbreviations: CI, confidence interval; HR, hazard ratio. ${ }^{a} n=472$ for logMAR VA analysis. ${ }^{\mathrm{b}} n=466$ for all OTS analyses. 
Table 4 Multivariate Cox proportional hazards regression of risk factors for traumatic glaucoma after open globe

\begin{tabular}{lccr}
\hline Risk factors & $H R$ & $95 \%$ CI & P-value \\
\hline Prior penetrating keratoplasty & 2.336 & $1.251-4.364$ & 0.008 \\
Zone II injury & 1.485 & $1.027-2.146$ & 0.036 \\
Vitreous hemorrhage & 2.181 & $1.279-3.719$ & 0.004 \\
Penetrating keratoplasty after open globe & 2.119 & $1.390-3.232$ & $<0.001$ \\
\hline
\end{tabular}

Abbreviations: CI, confidence interval; HR, hazard ratio.

Among patients with traumatic IOP elevation, the mean IOP at final follow-up was $16.2 \pm 6.9 \mathrm{~mm} \mathrm{Hg}$ on $0.9 \pm 1.2$ medications. Among patients with traumatic glaucoma, the mean IOP at final follow-up was $18.2 \pm 7.7 \mathrm{~mm} \mathrm{Hg}$ on $1.7 \pm 1.2$ medications; 25 patients (78.1\%) had IOP $<22 \mathrm{~mm} \mathrm{Hg}$ at last follow-up.

The median $\log$ MAR VA for the entire study cohort improved from an initial mean of $1.92 \pm 1.05$ (equivalent of counting fingers, or $\sim 2.5 / 200$ ) to $1.40 \pm 1.09$ (equivalent of $\sim 20 / 400$ ) at final follow-up.

\section{Discussion}

Eye injury remains a common and preventable cause of blindness throughout the world. ${ }^{15}$ The annual cost of work-related eye injury is estimated to be 1-3 billion dollars in the United States. ${ }^{16}$ Post-traumatic glaucoma is a serious complication of open-globe injuries, with the potential to render blind an already damaged eye. In our study cohort of 515 patients with a visually salvageable open-globe injury, the 12-month Kaplan-Meier risk for development of traumatic IOP elevation or traumatic glaucoma was quite high, at 32.4 and $11.0 \%$, respectively.

Girkin et $a l^{4}$ studied data from the United States Eye Injury Registry, and of 3627 patients with penetrating ocular trauma, 97 (2.67\%) developed traumatic glaucoma, defined as elevated IOP, or optic nerve or VF abnormalities consistent with glaucoma. This study was limited to two encounters, with initial and 6-month follow-up visits being reported using standardized forms. Significant risk factors for glaucoma development were increased age, lens injury, VA $<20 / 200$, and intraocular inflammation at baseline. Turalba et $a l^{8}$ reviewed 382 patients with open-globe injury and follow-up of at least 2 months, and found that the risk of developing traumatic IOP elevation, defined as IOP $\geq 22 \mathrm{~mm} \mathrm{Hg}$ at $>1$ visit or requiring treatment, was $17 \%$ after follow-up of 20.6 months. Risk factors included increased age, hyphema, lens injury, and Zone II injury.

In the present study, using the same definition for IOP elevation as Turalba et al, ${ }^{8}$ we found a similar percentage of patients with traumatic IOP elevation (23\%). We found traumatic glaucoma, defined as glaucoma medication required for at least 3 months, or having glaucoma surgery, to be strongly associated with PKP before open globe as well as the need for PKP after initial injury, a new finding that has not been previously reported in a similar cohort. Other significant risk factors for traumatic glaucoma in the present study echo the findings of other authors, including older age as a risk factor for traumatic IOP elevation, ${ }^{4,8}$ although it was not a risk factor for traumatic glaucoma; vitreous hemorrhage, ${ }^{4}$ likely because it is a marker for more severe trauma, and in addition ghost cell glaucoma is a known complication of vitreous hemorrhage in the setting of zonular damage, which is common in open-globe patients; and Zone II injury, ${ }^{8}$ which appears to be associated with greater compromise of aqueous outflow due to the anatomic relationships found in that area. We also found, as other authors have shown, ${ }^{17}$ that patients who required glaucoma surgery in this study required multiple intraocular surgeries for visual rehabilitation (mean 3.0 before the glaucoma procedure).

The OTS is a scoring system that provides useful prognostic information for eyes that have undergone trauma; a smaller OTS score is typically associated with a worse final VA and prognosis.7,13-15,17-19 Turalba et al ${ }^{8}$ used one part of the OTS classification, mechanism of injury, and found that blunt rupture was not a predictive factor in the development of elevated IOP compared with penetrating injuries. We were unable to duplicate this finding in a larger cohort, using proportional hazards regression. In addition, we used all elements of the OTS classification system and found no association with development of traumatic IOP elevation and glaucoma in multivariate analysis, though an association with smaller OTS values (worse trauma) was noted with both traumatic IOP elevation and traumatic glaucoma by univariate analysis.

The prevalence of traumatic IOP elevation and glaucoma after PKP is known to be elevated, ranging between 11 and $48 \%,{ }^{20-24}$ and is perhaps particularly elevated in eyes after penetrating eye injury. ${ }^{25}$ Mechanisms for IOP elevation after PKP include postoperative edema and inflammation compromising the trabecular meshwork, angle scarring, peripheral anterior synechiae, retained viscoelastics, and steroid response. The cumulative effects of the initial traumatic injury on aqueous outflow followed by additional damage that may accompany multiple other intraocular surgeries and PKP might contribute to development of elevated IOP in these eyes. Although the presence of PKP at the time of 
open-globe injury was itself significantly associated with our outcome measures, having PKP surgery after openglobe repair remained highly significant even when eyes with prior PKP were excluded from analysis. In addition, we excluded all eyes with known glaucoma from our study cohort, so those patients included in the study who had prior PKP did not have known glaucoma before their open-globe injury.

Our study has several limitations. Our follow-up was short (mean of 12.6 months, but median of 4.0 months); however we chose to include all patients regardless of length of follow-up, to allow use of Kaplan-Meier survival analysis, which provides the most accurate estimate of the true incidence of our outcome measures in the face of disparate follow-up periods. Traumatic IOP elevation and traumatic glaucoma were defined solely by IOP measures or treatments for IOP elevation, because most patients did not have quantitative optic nerve and/or visual field assessment during follow-up. In many patients, the ability to view the optic nerve was compromised by the ocular traumatic, which also limited the ability of many patients to perform visual field testing. Obtaining these measures was also affected in some patients by short follow-up and the clinical focus on treatment of other vision-threatening complications associated with ocular trauma. In addition, in this retrospective study we were not able to gather all clinically relevant data on all subjects because of incomplete data recording and non-standardized follow-up regimens. For example, precise information regarding the mechanism of glaucoma that patients developed was often not available. However, our findings of a high incidence of traumatic IOP elevation and glaucoma after open-globe injury highlight the importance of this complication for open-globe patients. Patients at higher risk include older patients, and those with vitreous hemorrhage, zone II injury, or PKP before open globe or having subsequent PKP. Further prospective study of glaucoma after openglobe repair, with clearly defined treatment algorithms and investigation of mechanisms, would be welcome.

\section{Summary}

What was known before

- Traumatic IOP elevation or glaucoma following blunt ocular injury have an overall incidence ranging from 2 to $43 \%$.

- Few large studies have evaluated traumatic IOP elevation and traumatic glaucoma after open-globe injury.

What this study adds

- Traumatic IOP elevation associated with age.

- Traumatic glaucoma associated with the prior PKP, need for subsequent PKP, initial vitreous hemorrhage, and Zone II injury.

\section{Conflict of interest}

The authors declare no conflict of interest.

\section{Acknowledgements}

Unrestricted departmental grant from Research to Prevent Blindness.

\section{References}

1 Negrel AD, Thylefors B. The global impact of eye injuries. Ophthalmic Epidemiol 1998; 5: 143-169.

2 De Leon-Ortega JE, Girkin CA. Ocular trauma-related glaucoma. Ophthalmol Clin North Am 2002; 15: 215-223.

3 Milder E, Davis K. Ocular trauma and glaucoma. Int Ophthalmol Clin 2008; 48: 47-64.

4 Girkin CA, McGwin Jr G, Morris R, Kuhn F. Glaucoma following penetrating ocular trauma: a cohort study of the United States Eye Injury Registry. Am J Ophthalmol 2005; 139: 100-105.

5 Sihota R, Kumar S, Gupta V, Dada T, Kashyap S, Insan R et al. Early predictors of traumatic glaucoma after closed globe injury. Arch Ophthalmol 2008; 126: 921-926.

6 Girkin CA, McGwin Jr G, Long C, Morris R, Kuhn F. Glaucoma after ocular contusion. J Glaucoma 2005; 14: 470-473.

7 Weichel ED, Colyer MH, Ludlow SE, Bower KS, Eiseman AS. Combat ocular trauma visual outcomes during operations Iraqi and Enduring Freedom. Ophthalmology 2008; 115: 2235-2245.

8 Turalba AV, Shah AS, Andreoli MT, Andreoli CM, Rhee DJ. Predictors and outcomes of ocular hypertension after openglobe injury. J Glaucoma 2014; 23(1): 5-10.

9 Lin MP, Eksioglu U, Mudumbai RC, Slabaugh MA, Chen PP. Glaucoma in patients with ocular chemical burns. Am J Ophthalmol 2012; 154(3): 481-485.

10 Bach M, Schulze-Bonsel K, Feltgen N, Burau H, Hansen L. Author response: Numerical imputation for low-vision states [electronic letter]. Invest Ophthalmol Vis Sci 2007. http://www.iovs.org/content/47/3/1236/reply.

11 Flynn-Evans EE, Tabandeh H, Skene DJ, Lockley SW. Circadian rhythm disorders and melatonin production in 127 blind women with and without light perception. J Biol Rhythms 2014; 29(3): 215-224.

12 Ross RD. Is perception of light useful to the blind patient? Arch Ophthalmol 1998; 116(2): 236-238.

13 Pieramici DJ, Sternberg Jr P, Aaberg Sr TM, Bridges Jr WZ, Capone Jr A, Cardillo JA et al. A system for classifying mechanical injuries of the eye (globe). The Ocular Trauma Classification Group. Am J Ophthalmol 1997; 123(6): 820-831.

14 Kuhn F, Maisiak R, Mann L, Mester V, Morris R, Witherspoon CD. The Ocular Trauma Score (OTS). Ophthalmol Clin North Am 2002; 15(2): 163-165.

15 Knyazer B, Levy J, Rosen S, Belfair N, Klemperer I, Lifshitz T. Prognostic factors in posterior open globe injuries (zone-III injuries). Clin Experiment Ophthalmol 2008; 36(9): 836-841.

16 McGwin Jr G, Hall TA, Xie A, Owsley C. Trends in eye injury in the United States, 1992-2001. Invest Ophthalmol Vis Sci 2006; 47: 521-527. 
17 Andreoli MT, Andreoli CM. Surgical rehabilitation of the open globe injury patient. Am J Ophthalmol 2012; 153(5): 856-860.

18 Yu WMC, Steel D. Visual outcome after open globe injury: a comparison of two prognostic models - the Ocular Trauma Score and the Classification and Regression Tree. Eye 2010; 24(1): 84-89.

19 Sobaci G, Akin T, Erdem U, Uysal Y, Karagül S. Ocular trauma score in deadly weapon-related open-globe injuries. Am J Ophthalmol 2006; 141(4): 760-761.

20 Oruçoglu F, Blumenthal EZ, Frucht-Pery J, Solomon A. Risk factors and incidence of ocular hypertension after penetrating keratoplasty. J Glaucoma 2014; 23(9): 599-605.

21 Ayyala RS. Penetrating keratoplasty and glaucoma. Surv Ophthalmol 2000; 45(2): 91-105.
22 Nguyen NX, Langenbucher A, Seitz B, Kuchle M.

[Frequency and risk factors of intraocular pressure increase after penetrating keratoplasty]. Klin Monbl Augenheilkd 2000; 217(2): 77-81.

23 Huber KK, Maier AK, Klamann MK, Rottler J, Özlügedik S, Rosenbaum $\mathrm{K}$ et al. Glaucoma in penetrating keratoplasty: risk factors, management, and outcome. Graefes Arch Clin Exp Ophthalmol. 2013; 251(1): 105-116.

24 Karadag O, Kugu S, Erdogan G, Kandemir B, Eraslan Ozdil $\mathrm{S}$, Dogan OK. Incidence of and risk factors for increased intraocular pressure after penetrating keratoplasty. Cornea 2010; 29(3): 278-282.

25 Suleiman Y, Amm M, Duncker GI, Nolle B. [Prognosis of corneal transplantation after penetrating eye injury]. Klin Monbl Augenheilkd 2004; 221(8): 658-673. 